\section{Energy Focus \\ NMR reveals unexpected defects in lithium-ion battery electrodes}

Tf our batteries do not behave the way Iwe expect, we have a problem. Such unpredictability can arise from many sources, including difficult-to-detect nanoscale defects in the materials of the batteries. In the August 11 issue of Chemistry of Materials (DOI: 10.1021/ acs.chemmater.5b01234; p. 5212), Michaël Deschamps of the University of Orléans and the National Centre for Scientific Research, along with a team of researchers from France, detail their work to locate and characterize these defect structures in the electrodes of lithiumion batteries.

The researchers' lithium-ion battery uses a $\mathrm{LiVPO}_{4} \mathrm{~F}$ electrode in which the material is intercalative, meaning that a mobile atomic species moves into the spaces between the $\mathrm{LiVPO}_{4} \mathrm{~F}$ layers. In general, atoms in intercalation materials can have any of several oxidation or spin states. The standard method of measuring these states leaves their local nature unresolved-thus, sufficiently small atomic features go undetected.

Deschamps and co-workers used nuclear magnetic resonance (NMR) to characterize the local atomic structures at the nanoscale in this material. Unexpectedly, they found paramagnetic lithium environments associated with crystallographic Li sites, and calculated that these environments accounted for as much as $20 \%$ of the Li content in the electrode. Furthermore, they applied a radio frequency $(\mathrm{RF})$ dipole-dipole-recoupling

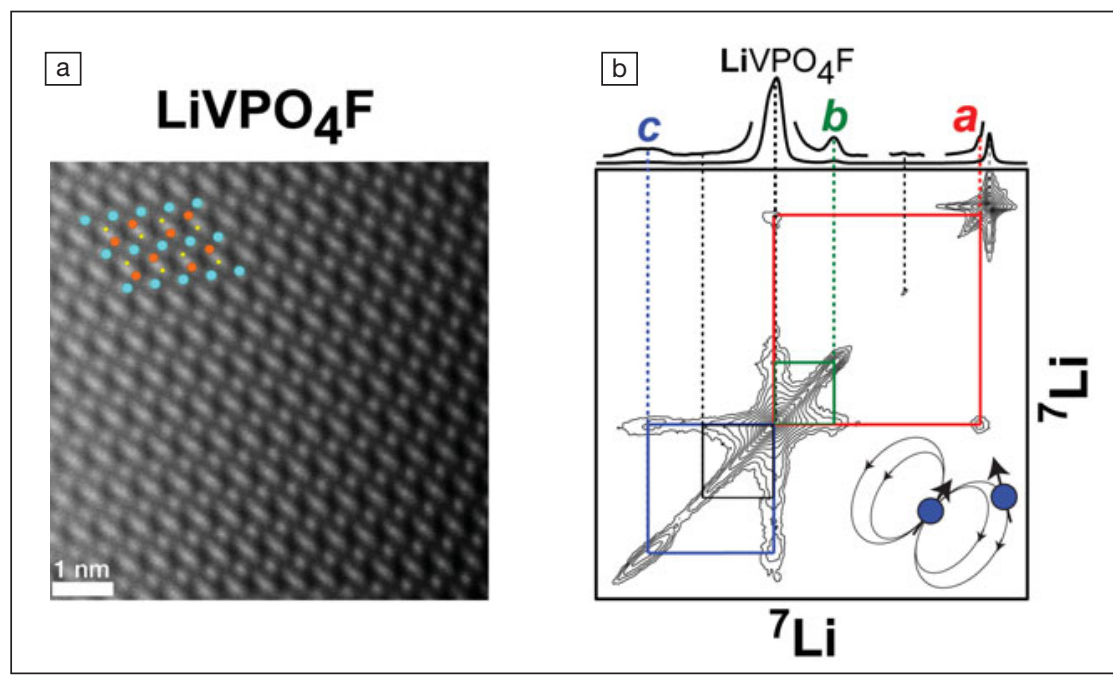

(a) Scanning transmission electron microscope image of phase-pure $\mathrm{LiVPO}_{4} \mathrm{~F}$ along the [010] crystal orientation. A LiVPO ${ }_{4} \mathrm{~F}$ unit cell is superimposed over the image, where vanadium, lithium, and phosphorus atoms are represented by blue, orange, and yellow spheres, respectively. (b) Solid lines represent correlated two-dimensional signals between crystallographic LiVPO ${ }_{4} \mathrm{~F}$ and the other paramagnetic ${ }^{7} \mathrm{Li}$ environments, establishing their respective ${ }^{7} \mathrm{Li}$ dipole-dipole interactions and hence subnanometer-scale proximities. Credit: Chemistry of Materials.

technique on a time scale roughly equal to the very short lifetimes of the atomic spin states, and registered enough of a signal for meaningful analysis. Combined with NMR spectra, the recoupling data allowed the researchers to determine unambiguously that these environments were associated with systemic structural defects in the crystal lattice, rather than representing a simple impurity phase. "These defects were completely undetected by other techniques, and we were really happy that NMR could show that there was more than meets the eye-or, in this case, the transmission electron microscope," Deschamps says.
In addition to the discovery of unexpected defects in a well-crystallized $\mathrm{LiVPO}_{4} \mathrm{~F}$ electrode, Deschamps and his colleagues demonstrated a new way to characterize certain kinds of paramagnetic materials: "One could imagine NMR spectroscopy being used to monitor the quality of materials, ensuring the batteries made with them will function as expected," Deschamps says. By characterizing the lithium-defect environments down to the atomic scale with remarkable precision, this work now provides researchers with a new, powerful tool to continue improving battery technology.

Antonio Cruz

\section{Bio Focus}

Synthetic biomaterials advance stem cell engineering

$\mathrm{T}$ he complexity and dynamism of biological tissues present a myriad of challenges for understanding these living composites, and for rebuilding them to benefit human health. Fortunately, this biological complexity can also serve as a boon to biomedical engineers by offering multifunctional cellular workhorses that are already predisposed to perform unique tasks. Arguably, the most valuable of such cellular tools are stem cells: systems which are naturally primed to form and repair human tissues. However, stem cells are so ripe with potential, being able to differentiate (i.e., gradually transform themselves) into many diverse tissue-specific cell types, that a key challenge in tissue engineering is learning how to direct stem cells to behave in predictable ways. As highlighted in a recent review article by $\mathrm{P}$. Chandra and S.-J. Lee of Wake Forest School of Medicine in Biomarker Insights (DOI: 10.4137/BMI.S20057; p. 105), innovations in biomaterial design and synthesis can play a significant role in enabling improved platforms for stem cell engineering. 
An ideal tissue-sculpting biomaterial would be able to mimic natural environmental niches that can coax stem cells into an appropriate series of responses along their tissue formation trajectory. These responses include adhesion, migration, proliferation, and differentiation (see Figure). In practice, designing the right environment is highly challenging, as native niches exhibit chemical, biological, and mechanical elements (e.g., small-molecule growth factors, signaling proteins, and extracellular matrix polymers) that contribute in concerted ways to direct stem cell fate. Progress, however, is being made, and Chandra and Lee survey this progressive research landscape.

Specifically, Chandra and Lee's review overviews and analyzes the results from more than 90 studies in which synthetic biomaterials have been applied to influence stem cell behavior. The authors

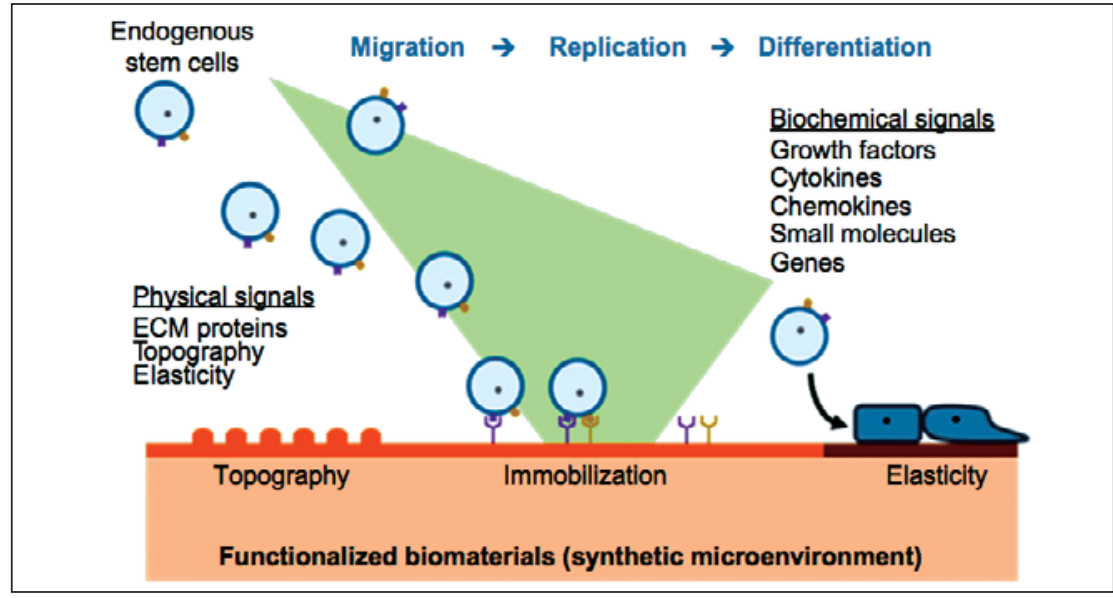

Schematic illustration of interactions between endogenous stem cells and synthetic microenvironment. Stem cells' fate in a particular microenvironment is regulated by intricate reciprocal molecular interactions with its surroundings. Credit: Biomarker Insights. discuss the roles of salient properties that affect a biomaterial's performance as an artificial niche, including biocompatibility, drug release behavior, mechanical elasticity, surface chemistry, and surface topography. Current favored materials show promise, but require further optimization. For example, electrospun polymers - such as poly(lactic-co-glycolide) — can be fabricated with tunable growth factor release kinetics, but the nanoscale morphological features of such materials are difficult to control reliably (thus hampering simultaneous control over the role of surface topography on stem cell response).

Due to the extensive combinatorial factors that influence stem cell proliferation and differentiation, the authors call for increased high throughput and computational studies as the field works toward a better understanding of extracellular matrix signals and their roles in controlling stem cell fate. However, despite the many lessons that remain ahead, the union of stem cells and engineered biomaterials holds great promise. Chandra and Lee emphasize this fact as they close their report by highlighting a variety of biomaterial-cell products that are currently under clinical development.

Lukmaan Bawazer

\section{Nano Focus}

Large-scale graphene gas barrier sets new record

raphene has been widely heralded $\checkmark$ as a revolutionary material for making thin barrier membranes among various other applications, but the experimental realization of graphene as a gas barrier has been limited. Although many research articles make reference to graphene's high barrier capabilities, very few experimental studies have verified this. "Everyone talks about it [graphene barriers], and nobody really questions the result. However, when you try to do it on a large scale, it doesn't really work at all," says researcher Christian Wirtz of Trinity College. That is, until now.
A recent article from Georg Duesberg's (Trinity College) laboratory, of which Wirtz is lead author, describes a novel large-scale graphene barrier that outperforms previously reported graphene barriers by a factor of 5000. The work is published in Advanced Materials Interfaces (DOI:10.1002/admi.201500082). Duesberg, Wirtz, and colleague Nina Berner (Trinity College) describe a highly effective oxygen gas barrier using stacks of chemical-vapor-deposited (CVD) graphene. A stack of three graphene layers $\left(\sim 5 \mathrm{~cm}^{2}\right.$ surface area) transmitted just $1.10 \times 10^{-17} \mathrm{~cm}^{3} \mathrm{~cm} / \mathrm{cm}^{2} \mathrm{~s}(\mathrm{~cm} \mathrm{Hg})$ of oxygen, or $1.1 \times 10^{-7}$ barrer, which is on par with most modern barriers, such as $\mathrm{AlO}_{x}$ or $\mathrm{SiO}_{x}$. The oxygen and moisture barrier properties of graphene, coupled with its size and high conductivity, make it an ideal candidate for a wide variety of applications, including flexible electronic displays and microelectronics packaging.

The graphene barrier was prepared by stacking CVD-grown graphene onto a $150-\mu \mathrm{m}$ polyethylene terephthalate (PET) substrate, a food packaging polymer that is known for good barrier properties. Unfortunately, "current CVD methods always have grain boundaries [defects] that give way to diffusion," Wirtz says. "We tried with just monolayer graphene and there was no improvement whatsoever. Then we started stacking it the right way. We got improvement, which was very exciting." The best barrier results came from a stack of three layers of CVD graphene, as shown in the graph. 